of iron always present in the raw materials cause a yellowish-brown discoloration with a lowering of the transparency of the body mixture.

The Langenthal kiln is about 100 metres long with two tunnels, one for biscuit firing at $900^{\circ} \mathrm{C}$. and the other for sharp firing at $1,400^{\circ} \mathrm{C}$. The working experience of this kiln shows that apart from the smaller maintenance charges and high percentage of first-class ware obtainable, the savings in saggars is much more than the replacement charges on the elements. The writer concludes by saying that the success of the electric kilns can only be based on a proper combination of electrical engineering and ceramic knowledge.

\title{
VIBRATION OF PROPELLER BLADES
}

$\mathrm{E}$ ARLY in 1936, at the instigation of the National Physical Laboratory, the Singing Propeller Committee of the Institution of Naval Architects included among other recommendations in its report a proposed research on "the manner in which typical blades vibrate and the effect on vibration characteristics of changes in shape, thickness, etc." Some two years later the research was approved. by the Advisory Committee of the William Froude Laboratory.

One of the difficulties involved in the discussion of vibrating propellers has been the absence of any experimental knowledge of the modes of vibration of propeller blades. A few experiments were made by Mr. Harry Hunter, using a shaped flat plate of uniform thickness which was set in motion by 'bowing' it. This gave some patterns, but not frequencies. Tests with a single propeller blade having a flat driving surface have recently been published by Kerr, Shannon and Arnold. As before, the blade was held in a vice and vibrated by bowing the edge. Frequencies measured by tuning fork and mono-chord are given with their diagrams.

The method adopted to vibrate the blades in the present experiments was devised in conjunction with the Physics Department of the National Physical Laboratory, the apparatus being made and the experiments being carried out by E. J. Evans of that Department.

The model propellers were one foot in diameter, made with two blades on a heavy boss $2 \cdot 25$ in. in diameter and $3 \cdot 2 \mathrm{in}$. in length. The blades were made flat on the driving face, which was in a horizontal plane when secured to the base plate by a 1.25 in. diameter bolt and nut. The propeller plates were made flat on the driving face, which was in a horizontal plane when secured to the base plate. The method of exciting vibration of the blades was by an electromagnet with its pole tips just above the blade surface. The pole tips were about $\frac{1}{8}$ in. square and $\frac{1}{8} \mathrm{in}$. apart, so that the exciting force was applied over a small area of the blade. The magnet could be moved horizontally over the whole surface of one blade. It was supplied with alternating current from a beat-tone oscillator and amplifier. The frequency could be varied continuously over a range of about 50-11,000 cycles per second. In addition to the alternating current, the magnet was supplied with direct current through a separate pair of coils. In this way the magnet produced an alternating field of known frequency superimposed on a direct field.

To find the resonant frequencies of the propelfer, fine sand was sprinkled on the upper surface of the blades, and the exciting current was slowly varied through a given and known range of frequency. The resonance frequencies were detected by the loudness of the note emitted by the propeller, and by the sand on the blades taking a definite pattern, the sand usually moving towards the nodal regions. The magnet was moved about the blade in the tip region until the elearest diagram had been obtained on the blade under the magnet and the most intense note had been found. The frequency was then noted, the magnet removed, its position marked by a black dot, and a photograph of the sand pattern taken. A wide selection of these photographs are used to illustrate the paper.

Since the work was undertaken with a general idea of the results being applied to the problem of the singing propeller, it was desirable to know the range of frequencies which need to be studied for this purpose. The noise (if any) made by a bronze propeller about $18 \mathrm{ft}$. in diameter, propelling a ship in smooth water, is either a low-pitched hum with beats in it proportional to the number of blades and a frequency varying from 200 cycles per second to a somewhat higher figure; or a grinding noise or grunt with a maximum once per revolution of propeller, the major tone of which may vary from 200 to 300 c.p.s. Either or both of these may be present.

There are two principal modes of vibration which need to be considered, namely, (1) that in which the blade tip as a whole moves perpendicularly to the blade surface, which is referred to as flexural, and (2) that in which the blade twists from the boss about a more or less central line lying on its surface, referred to as torsional. Formulæ for a blade of parabolic outline, obtained by Conn, are given for these two kinds of vibration.

Although the two blades of any propeller were made alike within the normal limits of error for model work, it was found that actually they had slightly different resonant periods. The extreme figures obtained for the two blades for any mode of vibration are given. It is concluded that some of the variations must arise from variations in the metal of the two blades. Torsional and mixed resonance are also discussed and tables of extreme values of cycles per second are given.

A broad comparison of the results obtained with those given by others indicates that most of the patterns obtained by $\mathrm{H}$. Hunter are at too high a frequency to have much bearing on the singing propeller problem. The patterns shown in the paper by Kerr, Shannon and Arnold present some difficulties when compared with those in this paper. Possibly the difference arises from the fact that in these tests the exciting force was continuous and not a single strong burst of vibration. Twenty-five vibration patterns of propeller blades are shown. 